\title{
Termoterapia en cáncer de próstata mediante el uso de nanopartículas magnéticas
}

\author{
Manfred Johannsen*, Uwe Gneveckow***, Kasra Taymoorian*, Chie Hee Cho**, \\ Burghard Thiesen**, Regina Scholz***, Norbert Waldöfner***, Stefan A. Loening*, \\ Peter Wust**, Andreas Jordan*** \\ *Department of Urology, Campus Mitte, Charité - Universitätsmedizin Berlin, Alemania. \\ **Department of Radiology, Campus Virchow-Klinikum, Charité - Universitätsmedizin Berlin, Alemania. \\ ${ }^{* * *}$ MagForce Nanotechnologies AG, Berlin, Alemania.
}

Actas Urol Esp. 2007;31(6):660-667

\section{RESUMEN}

\section{TERMOTERAPIA EN CÁNCER DE PRÓSTATA MEDIANTE EL USO DE NANOPARTÍCULAS MAGNÉTICAS}

En recientes ensayos clínicos, se ha evaluado en tumores malignos humanos, un nuevo método de dispensación de calor en pequeños espacios (intersticios) utilizando nanopartículas magnéticas y una técnica de inyección directa. En el cáncer de próstata, este procedimiento se ha investigado en dos estudios fase I separados empleando en uno solamente termoterapia de nanopartículas magnéticas y en otro en combinación con braquiterapia (implantes permanentes). En ambos estudios se demostró viabilidad y buena tolerancia, usando el primer prototipo de un aplicador de campo magnético. Como con cualquier otra técnica por calor, este nuevo procedimiento requiere herramientas específicas para su planificación, control de calidad y monitorización térmica, basado en una imagen apropiada y en técnicas de planificación. En estos primeros estudios, se evalúa un nuevo método que permite una planificación y distribución tridimensional no invasiva de la temperatura basado en la tomografia computerizada (TC). En la actualidad, los factores limitantes de este procedimiento son el malestar del paciente a altas intensidades de campos magnéticos y la distribución intratumoral subóptima de las nanopartículas. Hasta que estas limitaciones sean superadas y la termoablación pueda ser aplicada con seguridad como monoterapia, esta modalidad de tratamiento está siendo evaluada en combinación con la irradiación en pacientes con cáncer de próstata localizado.

Palabras clave: Termoterapia. Cáncer de próstata. Nanopartículas magnéticas.

\section{ABSTRACT}

THERMAL THERAPY OF PROSTATE CANCER USING MAGNETIC NANOPARTICLES

A novel method of interstitial heating using magnetic nanoparticles and a direct injection technique has been evaluated in human cancers in recent clinical trials. In prostate cancer, this approach was investigated in two separate phase-I-studies, employing magnetic nanoparticle thermotherapy alone and in combination with permanent seed brachytherapy. The feasibility and good tolerability was shown in both trials, using the first prototype of a magnetic field applicator. As with any other heating technique, this novel approach requires specific tools for planning, quality control and thermal monitoring, based on appropriate imaging and modelling techniques. In these first clinical trials, a newly developed method for planning and non-invasive calculations of the 3-dimensional temperature distribution based on computed tomography could be validated. Limiting factors of this approach at present are patient discomfort at high magnetic field strengths and suboptimal intratumoral distribution of nanoparticles. Until these limitations will be overcome and thermal ablation can safely be applied as a monotherapy, this treatment modality is being evaluated in combination with irradiation in patients with localized prostate cancer. 
$\mathrm{L}$ a hipertermia está aceptada como alternativa de tratamiento en monoterapia o junto a la radiación y/o a la quimioterapia para tratar el cáncer $^{1}$. Se sabe que un aumento moderado de la temperatura entre $40^{\circ} \mathrm{C}$ y $42-43^{\circ} \mathrm{C}$ aumenta los efectos tanto de la radio como de la quimioterapia, induciendo un cierto grado de apoptosis, mientras que temperaturas más altas tienen un efecto citotóxico directo sobre las células ${ }^{2}$. La aplicación de temperaturas por encima de $46^{\circ} \mathrm{C}$, se define como termoablación. Durante las últimas décadas, los tratamientos basados en el uso de la temperatura han sido ampliamente valorados tanto para la hiperplasia como en los procesos neoplásicos prostáticos ${ }^{3}$. Las técnicas de termoablación se han empleado como terapias de rescate tras fallos post-radioterapia o como tratamiento primario en pacientes seleccionados ${ }^{4,5}$.

A diferencia de la termoablación como monoterapia, durante mucho tiempo las estrategias se han dirigido hacia combinar la hipertermia con la radiación para explotar el conocido sinergismo terapéutico de estas dos modalidades en el tratamiento del cáncer de próstata ${ }^{6-11}$. Mejorando la dosis biológica de radiación con hipertermia, podemos conseguir que, con la misma eficacia, se administren dosis de radiación mas bajas, lo que reduce la toxicidad inducida por la radiación. Se ha demostrado in vitro e in vivo el efecto biológico de la termoterapia en el cáncer de próstata ${ }^{12}$. Sin embargo, el efecto clínico de la hipertermia en el cáncer de próstata es complejo debido a los tejidos circundantes y a la alta perfusión de este órgano. A menudo, las temperaturas eficaces en toda la próstata, se vieron limitadas por la elevación del campo eléctrico en los bordes. Estos fenómenos han limitado el hecho de alcanzar temperaturas y homogeneidad térmica con técnicas convencionales de calentamiento ${ }^{6-11}$. En consecuencia, se siguen buscando nuevas técnicas para la aplicación de hipertermia ${ }^{3}$, y todavía, la termoradioterapia para el cáncer de próstata no se está utilizando de forma rutinaria.

La termoterapia con nanopartículas magnéticas es una opción nueva, mínimamente invasiva, desarrollada para el tratamiento térmico intersticial $^{13}$. En esta técnica, se inyecta directamente en tumores superficiales o profundos, un fluido que contiene nanopartículas superparamagnéti- cas biocompatibles (fluido magnético), que son luego selectivamente calentadas en un campo magnético alterno. Esta tecnología es la primera técnica por calor que usa nanopartículas magnéticas, que ha sido sometida a ensayos clínicos. En este artículo se comunican los resultados actuales de la termoterapia mediante nanopartículas magnéticas en el cáncer de próstata y se esbozan las perspectivas futuras.

\section{TERMOTERAPIA CON NANOPARTÍCULAS MAGNÉTICAS: FUNDAMENTOS Y DATOS PRECLÍNICOS}

En contraste con los campos eléctricos en la hipertermia por radiofrecuencia, la interferencia del campo magnético con los tejidos sanos es, con ciertos limites, insignificante. Esto permite un calentamiento más selectivo de la masa objetivo por medio de hipertermia intersticial magnética. Las técnicas difieren en cuanto a la naturaleza y tamaño de los materiales magnéticos utilizados, con implicaciones como el índice de absorción de la energía, modo de deposición de la misma, aplicación y localización. Los materiales usados en la hipertermia mediada magnéticamente incluyen semillas (varillas de varios mm., de tamaño), partículas multidominio (1-300 $\mu \mathrm{m}$ ) y nanopartículas (1-100 nm), incluyendo estas últimas partículas subdominio (por debajo de 20 $\mathrm{nm}$ ). El calentamiento de un medio depende del índice específico de absorción (SAR) del implante en un campo magnético alterno. La energía es liberada al medio circundante como resultado de procesos físicos que varían de acuerdo al tamaño del material magnético utilizado y a la fuerza del campo magnético aplicado ${ }^{14}$. Mientras que los implantes más grandes, como las semillas, generan calor resistente a las corrientes circunferenciales inducidas en la superficie de las semillas por un campo magnético alterno, las partículas multidominio típicamente calientes por histéresis, pierden efecto. En contraste las nanopartículas y en especial las suspensiones de partículas subdominio generan calor principalmente a través de los procesos de relajación Brownian y Neel ${ }^{14}$.

Gilchrist en 1957 fue el pionero de la hipertermia con magnetitas pequeñas, más tarde desarrollada por Gordon et al. 20 años después ${ }^{14}$. 
La generalización en el uso clínico de esta técnica se vio entorpecida por limitaciones técnicas tales como incertidumbre con relación a la distribución intratumoral de las partículas y el calor. Además, en los estudios iniciales no se proporcionaba información sobre el efecto del calor en el crecimiento tumoral. Jordan et al. ${ }^{15}$ fueron los primeros en demostrar el potencial físico de las nanopartículas magnéticas con propósito de calentamiento y su superioridad sobre otros materiales de mayor tamaño. Las excelentes capacidades en cuanto al poder de absorción de los fluidos magnéticos en un campo magnético se pueden atribuir al gran número y enorme superficie de los elementos de calentamiento. Otra característica de las nanopartículas magnéticas es la hipertermia intracelular. Debido a su cubierta de tipo aminosilane, las nanopartículas (MFL AS, MagForce Nanotechnologies AG, Berlin, Alemania) son absorbidas intracelularmente mediante endocitosis diferencial ${ }^{16,17}$. Además la absorción selectiva de las mismas en las células tumorales prostáticas ha sido demostrada in vitro y ofrece la posibilidad de hipertermia selectiva de célula tumoral ${ }^{18}$. Los estudios en modelos múridos de carcinoma mamario, glioblastoma y cáncer de próstata han demostrado la viabilidad y eficacia de este método de calentamiento, así como una baja tasa de aclaramiento de las nanopartículas tumorales, permitiendo una serie de tratamientos con calor seguidos de una única inyección de fluido magnético ${ }^{19-21}$. En un modelo de tumor prostático se alcanzaron temperaturas termoablativas por encima de $70^{\circ} \mathrm{C}^{22}$. La viabilidad y eficacia de la terapia térmica combinada usando nanopartículas magnéticas y radiación se ha demostrado útil en un modelo de cáncer de próstata ani$\mathrm{mal}^{23}$.

\section{ESTUDIOS CLÍNICOS EN CÁNCER DE PRÓSTATA}

\section{Termoterapia mediante nanopartículas}

magnéticas como monoterapia

Objetivos del estudio y metodología

Se llevó a cabo un estudio fase I prospectivo para investigar la viabilidad de la termoterapia utilizando nanopartículas magnéticas en pacientes con cáncer de próstata localizado y con reci- diva local. Un pre requisito importante para la planificación y control de calidad del tratamiento en la terapia térmica es disponer de un método de imagen adecuado. La imagen generada mediante resonancia magnética (MRI) no se puede utilizar, dada la susceptibilidad de los artefactos, p.e. pérdida de la señal en las zonas de interes que contienen una alta concentración de nanopartículas de óxido de hierro. Utilizando la tomografía computarizada (TC), los depósitos de estas partículas aparecen a una densidad media de $450 \mathrm{HU}$, y pueden ser visualizados en el tejido prostático con una sensibilidad de alrededor del 90\% (Fig. 1) 24,25 . La viabilidad se definió como la capacidad de calentar la próstata, alcanzar la suficiente concentración intersticial de nanopartículas en la próstata en seis tratamientos secuenciales y evaluar un abordaje basado en TC para el control de calidad y realizar cálculos no invasivos de la distribución tridimensional de la temperatura. Otros objetivos fueron la toxicidad y la calidad de vida ( $\mathrm{OoL}$ ), evaluada usando los cuestionarios QLQ-C30 y QLQ-PR25 de la Organización Europea para la Investigación y el Tratamiento del Cáncer (EORTC). Los resultados oncológicos se valoraron mediante medición de las cifras séricas de PSA y la biopsia prostática.

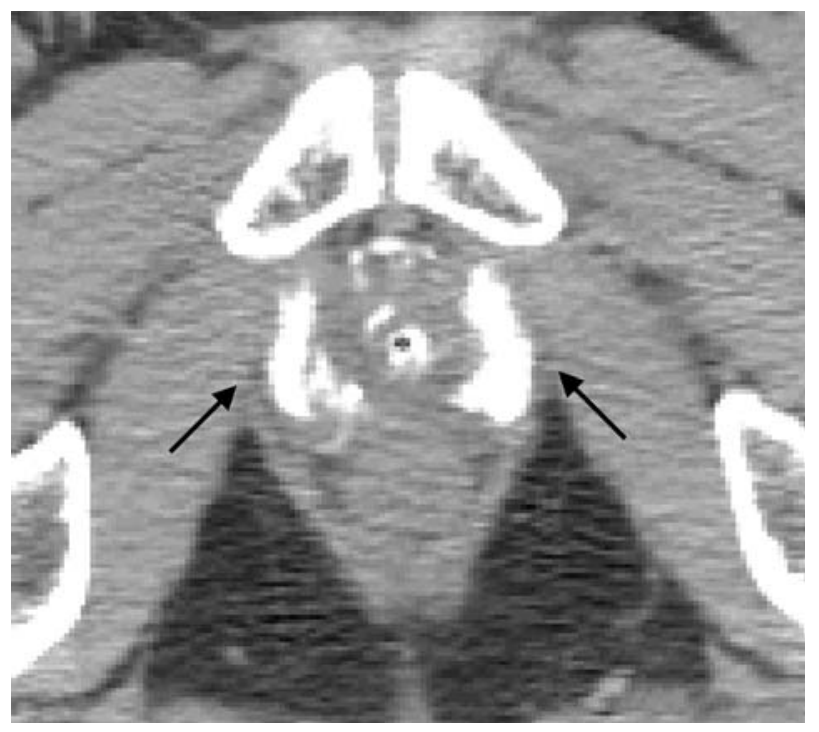

FIGURA 1. Ejemplo de una tomografia computarizada sin aumento obtenida después de la inyección de fluido magnético en la próstata, seguida de seis sesiones de termo terapia. Las flechas indican los depósitos hiperdensos de nanopartículas en la próstata. 
Se incluyeron diez pacientes (recidiva local y cáncer localizado), comprobado mediante biopsia. Tres pacientes tenían recurrencia local después de prostatectomía y radiación, los otros, recidiva tras radioterapia, andrógeno independiente en dos casos y hormono-refractario en uno de los casos. La dispersión de nanopartículas fue inyectada transperinealmente en la próstata bajo anestesia general y guiada con TRUS/fluorosco$\mathrm{pio}^{24}$. La termometría invasiva de la próstata fue realizada durante la primera y la última sesión, mientras que la termometría intraluminal en la uretra y en el recto se llevo a cabo en cada tratamiento. La distribución de la temperatura en la zona objetivo se calculó partiendo del volumen de hierro (obtenido del volumen de nanopartículas y la densidad en la TC), la fuerza del campo magnético durante los tratamientos y el SAR conocido del fluido magnético utilizado (asumiendo una perfusión constante), resolviendo la ecuación de la transferencia bio-calentamiento. Estos cálculos se correlacionaron con las cifras obtenidas en puntos de medida definidos de temperatura directa, para estimar la distribución tridimensional de la temperatura intraprostática en cada paciente ${ }^{25}$. Se administraron seis sesiones de termoterapia a intervalos semanales con el primer aplicador de campo magnético para uso en humanos (MFH300F, MagForce Nanotechnologies AG, Berlin (Fig. 2), usando un campo magnético alterno con una frecuencia de $100 \mathrm{kHz}$ y una fuerza de campo variable $(2,5-18 \mathrm{kA} / \mathrm{m})$.

\section{Viabilidad y medidas termales}

La planificación del tratamiento y el procedimiento de inyección fué viable en todos los pacientes. Sin embargo, no se pudo alcanzar la óptima (pre-calculada) distribución de las nanopartículas magnéticas en la zona objetivo debido a la resistencia mecánica del tejido pre-radiado de la próstata a la inyección de fluido magnético. El volumen medio de fluido aplicado fue de 11.4 $\mathrm{ml}(4.0$ - 14.0).

Se alcanzaron temperaturas máximas en la próstata de $55^{\circ} \mathrm{C}$. Las temperaturas medias en el 20,50 y $90 \%$ de las próstatas $\left(\mathrm{T}_{20}, \mathrm{~T}_{50} \mathrm{y} \mathrm{T}_{90}\right)$, bajo fuerzas de campo magnético constantes entre 4$5 \mathrm{kA} / \mathrm{m}$, que fueron toleradas durante todo el tratamiento por todos los pacientes, fueron 41,1

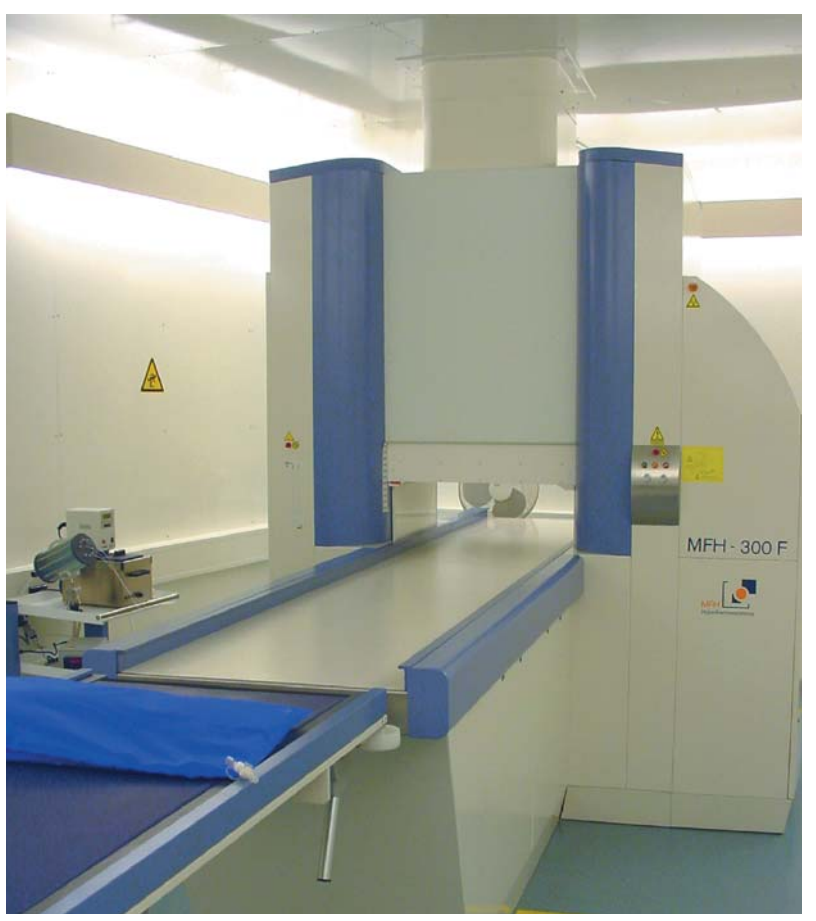

FIGURA 2. Aplicador de campo magnético alterno para uso en humanos (MFH3OOF, MagForce Nanotechnologies AG, Berlin) operando a una frecuencia de $100 \mathrm{kHz}$ y a una fuerza de campo variable $(0-18 \mathrm{kA} / \mathrm{m})$. Una unidad termométrica de fibra óptica y una consola de ordenador con software para monitorizar en linea la termoterapia, están conectadas al aplicador.

$(40,0-47,4), \quad 40,8 \quad(39,5-45,4) \quad$ y $\quad 40,1^{\circ} \mathrm{C} \quad(38,8-$ $43,4)$, respectivamente. La temperatura media uretral y rectal fueron $40,5^{\circ} \mathrm{C}(38,4-43,6) \mathrm{y}$ $39,8^{\circ} \mathrm{C}(38,2-43,4)$. La dosis media de temperatura derivada del ajuste entre medidas invasivas y no invasivas fue de 7.8 (3.5-136.4) minutos equivalentes acumulativos a $43^{\circ} \mathrm{C}$ en el $90 \%$ de las próstatas (CEM $43^{\circ} \mathrm{C} \mathrm{T}_{90}$; media: $20,9 \mathrm{~min}$ ). Se encontraron desviaciones entre la distribución de la temperatura simulada en las medidas invasivas cerca de la cara posterior de la vejiga y en la uretra (cálculos mas altos que las medidas directas) y en el periné (cálculos mas bajos que las medidas directas). En la próstata, no se encontraron diferencias apreciables entre los cálculos y las medidas directas ${ }^{25}$.

En las imágenes de la TC, los depósitos de las nanopartículas en la próstata fueron visibles hasta un año después del tratamiento termoterápico $^{26}$. Histológicamente también se detectaron nanopartículas en estos pacientes (Fig. 3). Sin embargo, no se intentó repetir el tratamiento. 


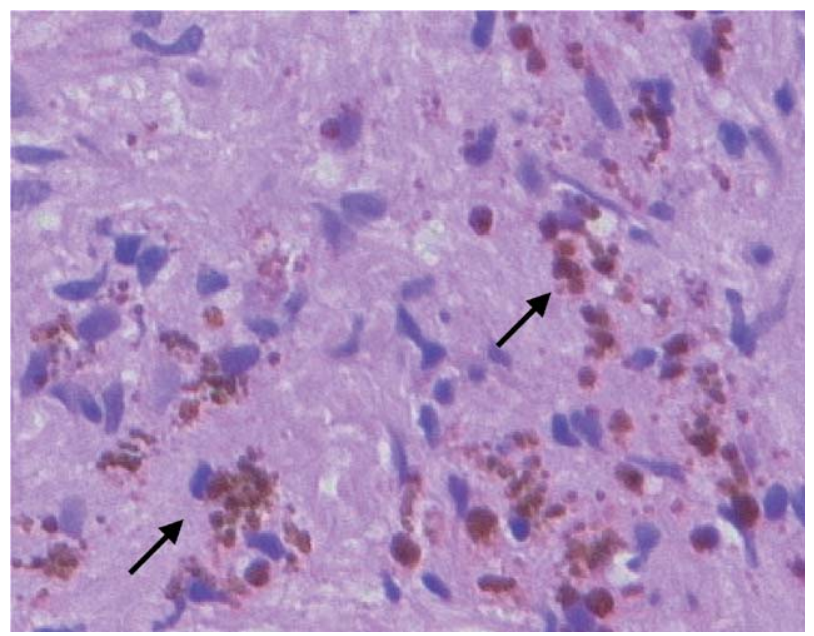

FIGURA 3. Imagen histológica obtenida de la biopsia de la próstata un año despues de la inyección de fluido magnético en la próstata y el tratamiento termoablativo. Las nanoparticulas de óxido de hierro (flechas) todavia están presentes en el tejido de la próstata (Tinción de hematoxilina-eosina x200).

Morbilidad, calidad de vida y resultados

No se observó ninguna toxicidad sistémica. Cuatro pacientes precisaron sondaje vesical debido a retención urinaria aguda, todos ellos tenían historia previa de obstrucción urinaria inferior tras radioterapia. Se observó toxicidad urinaria de grado 3 en dos pacientes, sólo después de la inyección de nanopartículas magnéticas y del subsiguiente primer tratamiento. Disuria de grado 2 en dos pacientes y de grado 1 en tres. Durante todo el tratamiento y todos los pacientes, toleraron fuerza alterna de campo magnético de $4-5 \mathrm{kA} / \mathrm{m}$. Valores mas altos de fuerza alterna magnética causaron molestias en la región inguinal y perineal. En algunos pacientes se observó una temperatura máxima de hasta $44^{\circ} \mathrm{C}$ a nivel de la epidermis, por lo general en los pliegues de las regiones escrotal y anal, pero se solucionaron mediante enfriamiento y ventilación. Aunque la intensidad del dolor en ambas zonas anatómicas estaba correlacionada con el incremento de la intensidad del campo magnético durante el tratamiento, no existió correlación directa entre esta toxicidad y las temperaturas alcanzadas en la próstata. No se observó en nuestro estudio, con un seguimiento medio de 10,5 meses (6-27), morbilidad postratamiento relacionada con el mismo $^{26}$.
Los cambios en la calidad de vida fueron evaluados durante y después del tratamiento comparándolos con los valores basales. No hubo deterioro importante en la forma física, estado de salud global y sintomas relacionados con el tratamiento durante el estudio ${ }^{26}$. Se observó un importante deterioro de la relación social $(p<0,0001)$, de la autoestima $(p=0,0008)$, fatiga $(p=0,0154)$, dolor $(p=0,0081)$, dificultades financieras $(p=0,0022)$, sintomas urinarios $(p=0,0445)$ y función sexual $(p=0,0096)$. De éstos, sólo la relación social permaneció afectada tres meses después del tratamiento. Dos pacientes se quejaron de empeoramiento en la función eréctil después de la termoterapia y recibieron tratamiento con inhibidores de la 5-fosfodiesterasa.

Se observó una disminución en las cifras de PSA en 8 pacientes al final del tratamiento $(>70 \%$ en un paciente, $40 \%$ en otro paciente $\mathrm{y} \leq 25 \%$ en el resto). La duración media de seguimiento mediante PSA fue de 5 meses (3-8). Tres pacientes tuvieron enfermedad a distancia y 7 pacientes presentaron progresión local durante el seguimiento.

\section{Termoterapia de nanopartículas magnéticas combinada con braquiterapia permanente}

\section{Material y Métodos}

Se estudió mediante un ensayo independiente fase-I, la viabilidad de combinar el procedimiento de termorradioterapia intersticial junto con la braquiterapia permanente. Se incluyó a ocho pacientes con fallo local tras radioterapia externa administrando termoterapia de nanopartículas magnéticas combinada con braquiterapia de baja tasa de dosis (LDR) (dosis prescripta 90-100 Gy), como tratamiento de rescate. Se procedió a una inyección de suspensión de nanopartículas como implantación permanente de semillas de I125, bajo anestesia general, guiada mediante TRUS y fluoroscopio. El objetivo de este procedimiento fue alcanzar una temperatura en la zona tumoral de $\mathrm{T}_{90}=42^{\circ} \mathrm{C}$, en base a los supuestos pre-planifi$\operatorname{cados}^{27}$.

Viabilidad y morbilidad de la termorradioterapia intersticial combinada

Los procedimientos de implante de semillas e inyección de fluido magnético fueron viables en 
todos los casos. La cantidad media de fluido magnético aplicada en este estudio fue de $8,5 \mathrm{ml}(6,0-$ 12,5). La $\mathrm{T}_{90}$ media alcanzada fue de $39,9^{\circ} \mathrm{C}$ $(38,8-42,3)$. Las temperaturas intraprostáticas máximas medidas y calculadas fueron de entre $42,4(40,6-45,5)$ y $41,5^{\circ} \mathrm{C}(40,9-45,4)$, respectivamente, mostrando una buena correlación entre medidas invasivas y cálculos no invasivos. La media CEM $43^{\circ} \mathrm{C} \mathrm{T}_{90}$ fue de 5,8 min (1-90) en esta serie de pacientes ${ }^{27}$.

Solo se observó toxicidad local. Dos pacientes presentaron dolor perineal persistente de grado 1-2 durante varios meses, probablemente mas relacionado con el implante de semillas que con el fluido magnético. Otro paciente tuvo que ser sometido a dos resecciones transuretrales en una institución diferente, seis y nueve meses despues de la implantación de las semillas, debido a obstrucción urinaria inferior. Este paciente tuvo, un año despues del procedimiento combinado de semillas permanentes y termoterapia una fistula rectal, por lo que necesitó una colostomía temporal. Los datos de los resultados oncológicos no están aún disponibles para este estudio.

\section{DISCUSIÓN Y PERSPECTIVAS FUTURAS}

La hipertermia clínica en el cáncer de próstata sigue siendo un reto. La próstata está protegida en su cara anterior por tejido adiposo y óseo y rodeada posterior y cranealmente por órganos huecos que contienen aire y fluido. Las diferentes conductividades de estos tejidos a la radiofrecuencia o a las ondas ultrasónicas aplicadas con el propósito de calentamiento, pueden causar reflexión no deseada, dispersión o absorción de la energía térmica fuera del área objetivo en zonas críticas, mientras que la perfusión intra y peri prostática puede actuar como un reservorio de calor. Todos estos fenómenos hacen que las temperaturas alcanzables en la próstata sean limitadas, con técnicas de calentamiento convencionales ${ }^{6-11}$.

En los presentes estudios, demostramos que la termoterapia de nanopartículas magnéticas es viable y que las temperaturas hipertermicas y termoablativas pueden alcanzarse en la próstata con fuerzas de campo relativamente bajas de 4-5
$\mathrm{kA} / \mathrm{m}^{24-27}$. Fuerzas de campo mas altas pueden causar malestar local, originados desde fenómenos distintos independientes de la presencia de nanopartículas, principalmente derivados de los efectos en los límites entre tejidos de diferente constante dieléctricas y de la conductividad, lo que puede suceder entre las superficies óseas y los tejidos blandos, y se incrementa a nivel epidérmico, particularmente en los pliegues, lo que puede conducir a puntos relativamente calientes. Estos efectos se observaron sólo durante el tratamiento de tumores pélvicos. De hecho, las fuerzas de campo magnético toleradas durante la termoterapia de nanopartículas magnéticas de tumores craneales fueron mucho mas altas, e.j. $10-14 \mathrm{kA} / \mathrm{m}^{28}$. Dado que con el aplicador utilizado en este estudio se pueden aplicar fuerzas de campo de hasta $18 \mathrm{kA} / \mathrm{m}$ y que el aumento cuadrático del índice específico de absorción (SAR) proporciona una fuerza de campo magnético aumentada, con esta técnica se podrían alcanzar, temperaturas significativamente más altas. También incrementando la homogeneidad de la distribución intraprostática de las nanopartículas, se podrían obtener gradiantes de temperatura más bajos y temperaturas mínimas más altas en la región anatómica concreta ${ }^{27}$.

Con respecto a los resultados oncológicos, se observó en ambos estudios que el PSA disminuyó después de la termoterapia, lo que sugiere eficacia potencial de este tratamiento. Sin embargo, las respuestas en el ensayo de monoterapia fueron de limitada extensión y duración. Debido a lo heterogéneo de la cohorte de pacientes, incluyendo pacientes con múltiples pre-tratamientos y con mayoritariamente desfavorables pronósticos, no es posible dar una interpretación con significado de la eficacia oncológica de este estudio en Fase I.

Los resultados de nuestro trabajo así como los ensayos previos realizados con animales sugieren que nuestra técnica puede, en principio, ser adecuada para la termoablación tanto en monoterapia o hipertermia en combinación con radioterapia en el cáncer de próstata. Sin embargo, dado que en la actualidad el promedio de temperaturas alcanzadas en la próstata con nuestra técnica son hipertérmicas, la combinación con radiación está siendo valorada en estos momentos. Desde 
el punto de visto radio-biológico, la aplicación simultánea de calor y radiación es considerada ideal para la termorradioterapia ${ }^{29}$. En concreto, el concepto de combinar la hipertermia suave con la radiación LDR ha sido propuesto de manera prometedora, pero, que nosotros sepamos nunca se ha llevado a cabo clínicamente ${ }^{30}$. Con este fin, hemos comenzado en nuestra institución un estudio de Fase II en pacientes con cáncer de próstata clínicamente localizado y con criterio de riesgo intermedio (PSA 10-20ng/ml o suma de Gleason 7), que no habían sido tratados previamente. En este ensayo, los pacientes recibieron braquiterapia LDR (semillas de I125, dosis prescripta 145 Gy) combinada con termoterapia de nanopartículas magnéticas. Los criterios de valoración son tasa de control local del tumor, toxicidad y calidad de vida. Los primeros resultados de este estudio se esperan para dentro de 3-5 años.

Se han iniciado en nuestros laboratorios estudios adicionales relativos a la aplicación sistémica de nanopartículas. Estos estudios se focalizaran en los mecanismos y objetivos pasivos y activos de las nanopartículas para dirigirlas hacia las células tumorales después de su administración sistémica.

\section{CONCLUSIONES}

El calentamiento intersticial usando nanopartículas fue viable en pacientes con cáncer de próstata con recidiva local y que habían sido previamente radiados. La toxicidad relacionada con el tratamiento fue moderada y la calidad de vida se afectó sólo temporalmente. Los factores limitantes de esta técnica en el momento actual son las molestias producidas por las fuerzas de campo magnético altas y la distribución intratumoral subóptima. Por otra parte, los depósitos de nanopartículas en las próstatas son muy duraderos.

En el momento actual no existe un sistema disponible ni para la inyección directa de fluido magnético bajo control visual a tiempo real ni tampoco para una imagen fiable del tumor dentro de la próstata. Por tanto, la ablación selectiva del cáncer de próstata separando el tejido normal no es posible en este momento, pero puede llegar a ser un tema interesante en el futuro con técni- cas de diagnóstico de imagen mejoradas. Hasta que nuestro método de calentamiento esté más depurado y se puedan aplicar con seguridad fuerzas de campo magnético más altas para conseguir un mayor rendimiento en monoterapia, esta modalidad de tratamiento está siendo valorada en combinación con la radiación en pacientes con cáncer de próstata localizado.

\section{Agradecimientos}

Los estudios fueron realizados con el apoyo del Proyecto EFRE "NanoMed", Nanotecnologia en Medicina, No. 2000-22006 2ue/2 y en parte por la Fundación Lieselotte-Beutel (Centro Proyecto Próstata). Los autores agradecen al Dr. Florian Fritzsche, Departamento de Patologia, Charité-Universitätsmedizin Berlín, por las imágenes obtenidas de las biopsias de próstata.

\section{REFERENCIAS}

1. Wust P, Hildebrandt B, Sreenivasa G, Rau B, Gellermann J, Riess H, Felix R, Schlag PM. Hyperthermia in combined treatment of cancer. Lancet Oncol 2002;3(8):487-497.

2. Hildebrandt B, Wust P, Ahlers O, Dieing A, Sreenivasa G, Kerner T, Felix R, Riess H. The cellular and molecular basis of hyperthermia. Crit Rev Oncol Hematol. 2002;43 (1):33-56.

3. Stauffer PR. Evolving technology for thermal therapy of cancer. Int J Hyperthermia. 2005;21(8):731-744.

4. Touma NJ, Izawa JI, Chin, JL. Current status of local salvage therapies following radiation failure for prostate cancer. J Urol 2005;173(2):373-379.

5. Colombel M, Poissonier L, Martin X, Gelet A. Clinical results of the Prostate HIFU Project. Eur Urol. 2007;5 (Suppl 6):491-494.

6. Van Vulpen M, De Leeuw AA, Raaymakers BW, Van Moorselaar RJ, Hofman P, Lagendijk JJ, Battermann JJ. Radiotherapy and hyperthermia in the treatment of patients with locally advanced prostate cancer: preliminary results. BJU Int. 2004;93(1):36-41.

7. Anscher MS, Samulski TV, Dodge R, Prosnitz LR, Dewhirst MW. Combined external beam irradiation and external regional hyperthermia for locally advanced adenocarcinoma of the prostate. Int $\mathrm{J}$ Radiat Oncol Biol Phys. 1997; 37(5):1059-1065.

8. Hurwitz MD, Kaplan ID, Hansen JL, Prokopios-Davos S, Topulos GP, Wishnow K, Manola J, Bornstein BA, Hynynen K. Hyperthermia combined with radiation in treatment of lovally advanced prostate cancer is associated with a favourable toxicity profile. Int $J$ Hyperthermia. 2005;21(7):649-656.

9. Algan O, Fosmire H, Hynynen K, Dalkin B, Cui H, Drach G, Stea B, Cassady JR. External beam radiotherapy and hyperthermia in the treatment of patients with locally advanced prostate carcinoma. Cancer. 2000;89(2):399403.

10. Kalapurakal JA, Pierce M, Chen A, Sathiaseelan V. Efficacy of irradiation and external hyperthermia in locally advanced, hormone-refractory or radiation recurrent prostate cancer: a preliminary report. Int $J$ Radiat Oncol Biol Phys 2003;57(3):654-664. 
11. Tilly W, Gellermann J, Graf R, Hildebrandt B, Weißbach L, Budach V, Felix R, Wust P. Regional hyperthermia in conjunction with definitive radiotherapy against recurrent or locally advanced prostate cancer T3 pNO MO. Strahlenther Onkol 2005;181(1):35-41.

12. Ryu S, Brown SL, Khil MS, Kim SH, Kim JH. Preferential radiosensitization of human prostatic carcinoma cells by mild hyperthermia. Int $J$ Radiat Oncol Biol Phys. 1996;34(1):133-138.

13. Jordan A, Maier-Hauff K, Wust P, Johannsen M. Nanoparticles for thermotherapy. In: Kumar CSSR, editor. Nanomaterials for cancer therapy. Weinheim: Wiley-VCH, 2006;242-258.

14. Jordan A, Scholz R, Wust P, Fähling H, Wust P. Magnetic Fluid Hyperthermia (MFH) : Cancer treatment with AC magnetic field induced excitation of biocompatible superparamagnetic nanoparticles. J Magn Magn Mater. 1999; 201:413-419.

15. Jordan A, Wust P, Fähling H, John W, Hinz A, Felix R. Inductive heating of ferrimagnetic particles and magnetic fluids: physical evaluation of their potential for hyperthermia. Int J Hyperthermia 1993;9(1):51-68.

16. Jordan A, Wust P, Scholz R, Tesche B, Fähling H, Mitrovics T, Vogl T, Cervos-Navarro J, Felix R. Cellular uptake of magnetic fluid particles and their effects in AC magnetic fields on human adenocarcinoma cells in vitro. Int $\mathrm{J}$ Hyperthermia. 1996;12,(6):705-722.

17. Jordan A, Scholz R, Wust P, Schirra H, Schiestel T, Schmidt H, Felix R. Differential Endocytosis of dextran and silan-coated magnetite nanoparticles and the effect of intracellular hyperthermia on human mammary carcinoma cells in vitro. J Magn Magn Mater 1999;194:185-196.

18. Jordan A, Scholz R, Maier-Hauff K, Johannsen M, Wust P, Nadobny J, Schirra H, Schmidt H, Deger S, Loening SA, Lanksch W, Felix R. Presentation of a new magnetic field therapy system for the treatment of human solid tumors with magnetic fluid hyperthermia. J Magn Magn Mater 2001;225:118-126.

19. Jordan A, Wust P, Scholz R, Mitrovics T, Fähling H, Gellermann J, Vogl T, Cervos-Navarro J, Felix R. Effects of Magnetic Fluid Hyperthermia (MFH) on C3H mammary carcinoma in vivo. Int $\mathrm{J}$ Hyperthermia 1997;13(6):587605.

20. Jordan A, Scholz R, Maier-Hauff K, Van Landeghem FKH, Waldöfner N, Teichgräber U, Pinkernelle J, Bruhn H, Neumann F, Thiesen B, Von Deimling A, Felix R. The effect of thermotherapy using magnetic nanoparticles on rat malignant glioma. J Neurooncol. 2006;78(1):7-14.

21. Johannsen M, Jordan A, Scholz R, Lein M, Koch M, Deger S, Roigas J, Jung K, Loening SA. Evaluation of magnetic fluid hyperthermia in a standard rat model of prostate cancer. J Endourol 2004;18(5):495-500.
22. Johannsen M, Thiesen B, Jordan A, Taymoorian K, Gneveckow U, Waldöfner N, Scholz R, Koch M, Lein M, Jung K, Loening SA. Magnetic fluid hyperthermia (MFH) reduces prostate cancer growth in the orthotopic Dunning R3327 rat model. Prostate 2005;64(3):283-292.

23. Johannsen M, Thiesen B, Gneveckow U, Taymoorian K, Waldöfner N, Scholz R, Deger S, Jung K, Loening SA, Jordan A. Thermotherapy using magnetic nanoparticles combined with external radiation in an orthotopic rat model of prostate cancer. Prostate 2006;66(1):97-104.

24. Johannsen M, Gneveckow U, Eckelt L, Feussner A, Waldöfner N, Scholz R, Deger S, Wust P, Loening SA, Jordan A. Clinical hyperthermia of prostate cancer using magnetic nanoparticles: presentation of a new interstitial technique. Int J Hyperthermia 2005;21:637-647.

25. Johannsen M, Gneveckow U, Thiesen B, Taymoorian K, Cho CH, Waldöfner N, Scholz R, Jordan A, Loening SA Wust P. Thermotherapy of Prostate Cancer Using Magnetic Nanoparticles: Feasibility, Imaging and Three-Dimensional Temperature Distribution. Eur Urol 2006 Nov 17; [Epub ahead of print].

26. Johannsen M, Gneveckow U, Taymoorian K, Thiesen B, Waldöfner N, Scholz R, Jung K, Jordan A, Wust P, Loening SA. Morbidity and quality of life during thermotherapy using magnetic nanoparticles in locally recurrent prostate cancer: results of a prospective phase I trial. Int $\mathrm{J}$ Hyperthermia 2007 23(3):315-323.

27. Wust P, Gneveckow U, Johannsen M, Böhmer D, Henkel T, Kahmann F, Sehouli J, Felix R, Ricke J, Jordan A. Magnetic nanoparticles for interstitial thermotherapy - feasibility, tolerance and achieved temperatures. Int $\mathrm{J}$ Hyperthermia 2006;22(8):673-85.

28. Maier-Hauff K, Rothe R, Scholz R, Gneveckow U, Wust P, Thiesen B, Feussner A, Von Deimling A, Waldöfner N, Felix R, Jordan A. Intracranial Thermotherapy using Magnetic Nanoparticles Combined with External Beam Radiotherapy: Results of a Feasibility Study on Patients with Glioblastoma Multiforme. J Neurooncol 2007;81(1):53-60.

29. Kampinga HH, Dikomey E. Hyperthermic radiosensitization: mode of action and clinical relevance. Int $\mathrm{J}$ Radiat Biol 2001;77(4):399-408.

30. Armour EP, Raaphorst GP. Long duration mild temperature hyperthermia and brachytherapy. 30. Int J Hyperthermia. 2004;20(2):175-89.

Correspondencia autor: Dr. Manfred Johannsen

Department of Urology, Campus Mitte.

Charité-Universitätsmedizin Berlin.

Charitéplatz, 1 - 10117 Berlín (Alemania)

Tel.: 0049-30-450615163

E-mail autor: manfred.johannsen@charite.de

Información artículo: Original 